\title{
Virtual Design and Visual Simulation of Cathode Target on Magnetron Sputtering Coater
}

\author{
Yichen Zhang ${ }^{1, a}$, Canlun $\mathrm{Li}^{1, \mathrm{~b}}$, Xinying $\mathrm{Li}^{1, \mathrm{c}}$, Hui $\mathrm{Li}^{1, \mathrm{~d}}$ \\ ${ }^{1}$ School of Mechanical Engineering \& Automation, Northeastern University, Shenyang 110819 \\ China \\ aychzhang@me.neu.edu.cn, '062licanlun@163.com, clixinying_0412@126.com, \\ dihui881115@163.com
}

Keywords: Magnetron Sputtering coater, Virtual design, Visual simulation

\begin{abstract}
In order to solve the traditional problems such as long designing cycle and high designing cost, the research applies the virtual simulation technology to the design of cathode target on magnetron sputtering vacuum coater. Through analyzing, modeling and simulating, the process model of a typical cathode target on magnetron sputtering coater is proposed. The virtual design of cathode target framework based on distributed collaborative simulation is constructed, which provides a theorial basis for the research of virtual design on cathode target.Using Solidworks software, parts modeling and assembly modeling of cathode target are realized. Using ADAMS, the movements of charged particles in magnetic field and high frequency alternating electric field are simulated, and the visual animation simulation of particles movement is achieved. The research demonstrates the feasibility of virtual simulation technology on vacuum coater design.
\end{abstract}

\section{Introduction}

Magnetron sputtering coater is widely used in industrial plating for its high-speed, low temperature and other characteristics. With the development of science and technology, the industrial application requests a significant exaltation in parameters, such as film uniformity and thin film adhesion etc.. However, The design of domestic magnetron sputtering coater usually adopts traditional design mode, and designers mainly depends on their old experiences in the design, so it is quite difficult to achieve designed effect by the traditional designing method.

The virtual prototyping technology is applied to the design of the magnetron sputtering coater in order to solve the problems in the traditional designing, such as long designing time, high designing cost and low film quality. Virtual simulation technology is a digital designing method based on the simulated models of the products, and it can help the designers to realize the modifications, characteristics testing, manufacturing simulation and operation simulation in the virtual environment $[1,2,3,4,5]$. The paper takes the design process of cathode target on magnetron sputtering coater for example to perform early research and demonstrates the feasibility of vacuum coater virtual design.

\section{Virtual design of cathode target}

Virtual design of cathode target on magnetron sputtering coater uses virtual model to simulate the design, manufacture, installation, operation and maintenance to obtain the optimized performance. In this process, the virtual model is the basis of virtual simulation, and is used from the beginning of conceptual design to final manufacturing analysis. In fact, the development process of virtual model 
also can be regarded as a continual refining and improving process. In this process, the model can be gradually established from coarse particle size to fine grit, and the design, manufacture, installation, operation and maintenance can be completed. Virtual design uses accurate mathematical model which includes geometry, transmission and connection, physical characteristics, beam dynamics etc. to express each parts, each components and overall coater.

In this paper, Solidworks software is used as modeling tools to realize the design process cathode target, and the process includes parts modeling, assembly modeling and the drawing of engineering drawings. Fig. 1 shows the general design process of virtual design.

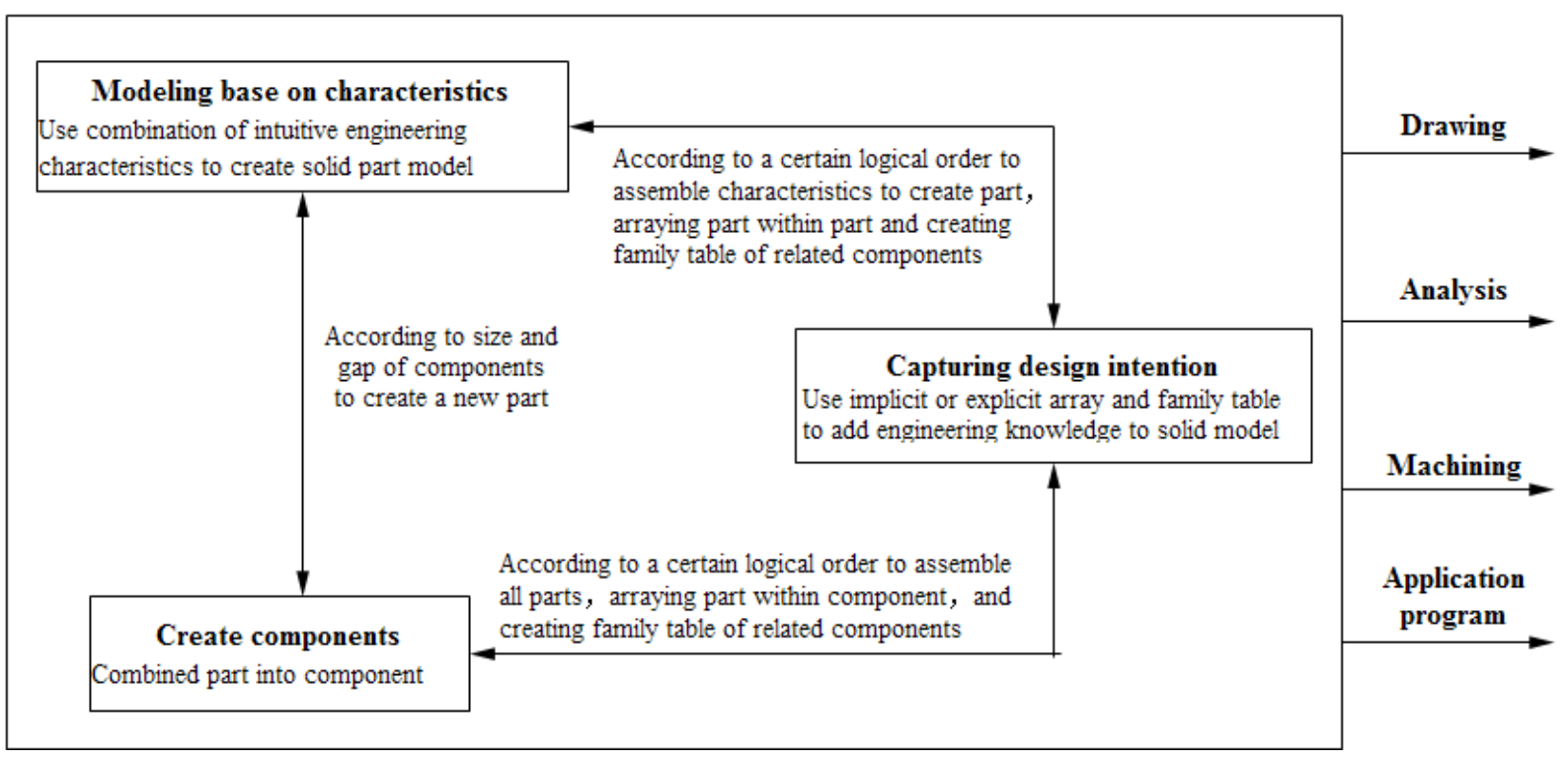

Fig. 1. Modeling flow of virtual design

Virtual parts modeling. The most basic components of assembly model is parts. Using Solidworks software, all the parts models are gained. Fig. 2 shows the characteristics expression of magnetic conductive plate on cathode target.

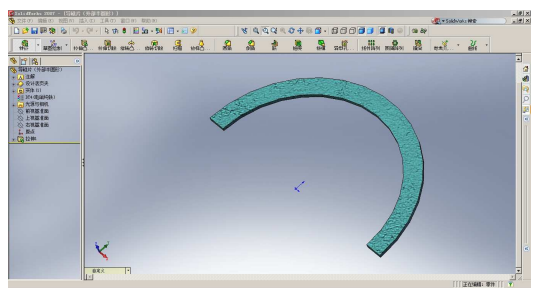

Fig. 2. Characteristics expression of magnetic conductive plate

Virtual assembly of parts. The general process of assembling is to open a new assembly file firstly, then input the main parts or components into assembly file and put the parts or components into suitable site through the rotation and input the secondary parts or components into assembly file, and use mating function to add constraints to the parts or components for fixing site at last. According to these steps, all parts or components are inputed into assembly file and added constraints to form the whole model ultimately. Fig. 3 shows the assembly process of cathode target, and Fig.4 shows the 3D model of cathode target on magnetron sputtering coater. 


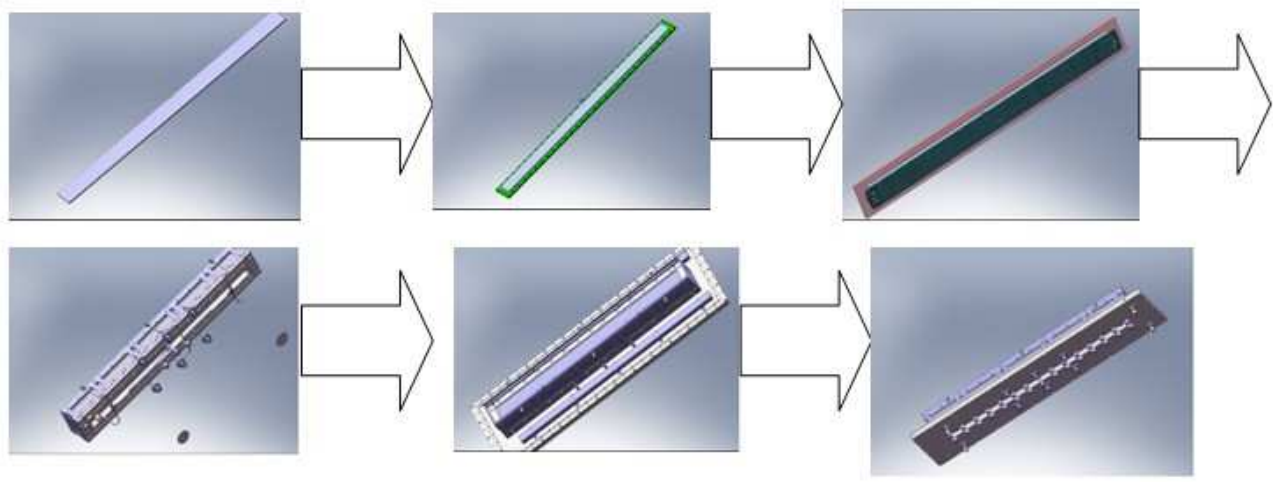

Fig. 3. The assembly process of cathode target

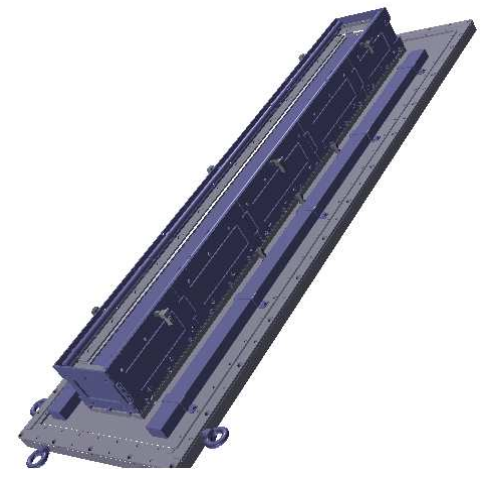

Fig. 4. Cathode target assembly model

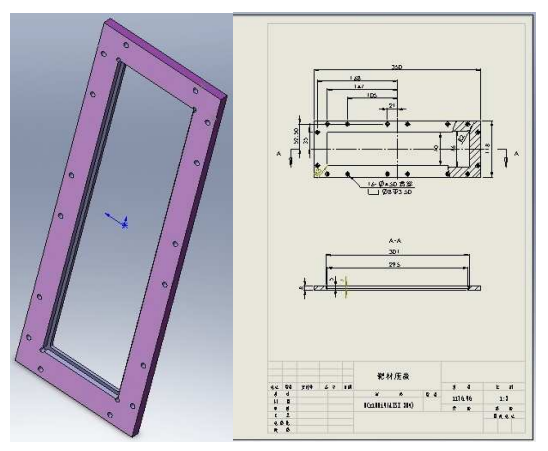

Fig. 5. 2D drawing of gland bush from 3D model

The drawing of engineering drawings. The $2 \mathrm{D}$ engineering drawing is used to conduct the produce and manufacture, which can be acquired through the projection of 3D solid model from different angle after the establishment of 3D solid model. Moreover, because of the full correlation of Pro/Engineer Software, any changes in 2D drawings are automatically reflected in the 3D parts and assembly model and vice versa. Fig. 5 shows $2 \mathrm{D}$ engineering drawing of gland bush from 3D model on magnetron sputtering cathode target.

\section{Visual simulation of charged particles}

The visual simulation of cathode target is mainly focused on the visual simulation of particles. First, magnet field and electric field generated by RF power are analyzed. On this basis, dynamics calculations of the particles are made. Finally, the result of dynamics calculation is used to visualization of particle motion for evaluating the design of magnetron sputtering coater. The purpose of visualization is to explore a way which inducts physical process into magnetron sputtering coater design process for helping designers to intuitively understand the acceleration process and evaluate the design effect.

In the analysis of magnetic field, considering the nonlinearity of magnetic field, ANSYS software is used to analyze 2D magnetic field [6,7,8], as shown in Fig. 6. 

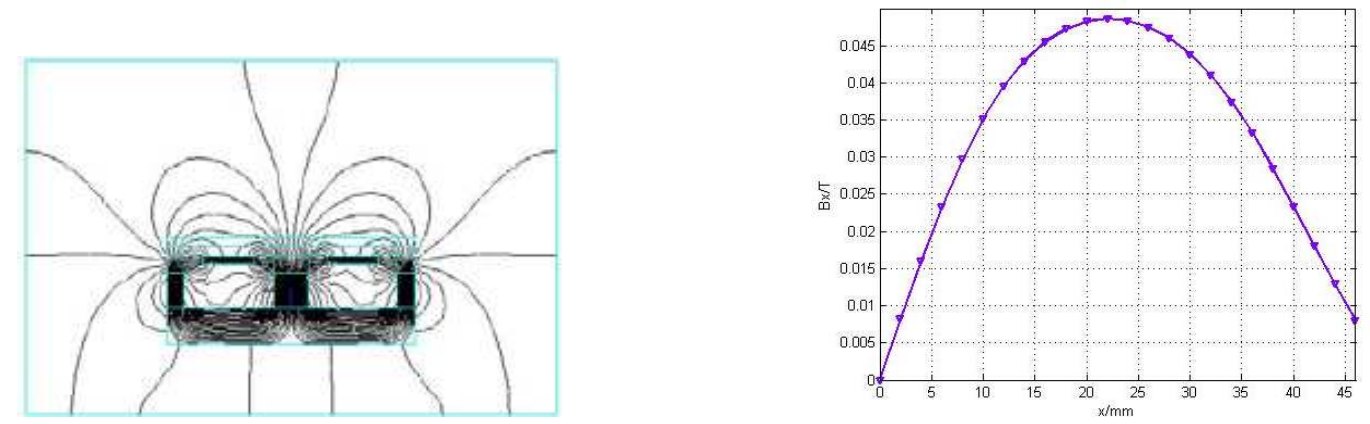

(a) magnetic line distribution of middle section (b)horizontal magnetic induction intensity of target

Fig. 6. 2D magnetic line distribution cathode target process

Also the physical dimension of parts or components in target is changed to alter the distribution of horizontal magnetic induction intensity. For example, the heights of inner and outer magnets are changed in order to change the distribution of horizontal magnetic induction intensity for optimizing the distribution. The simulation result is shown as Fig. 7. From the simulation result, the distribution of horizontal magnetic induction intensity has relatively good uniformity when the height of inner magnets is about $19 \mathrm{~mm}$ and the height of outer magnets is about $20 \mathrm{~mm}$.

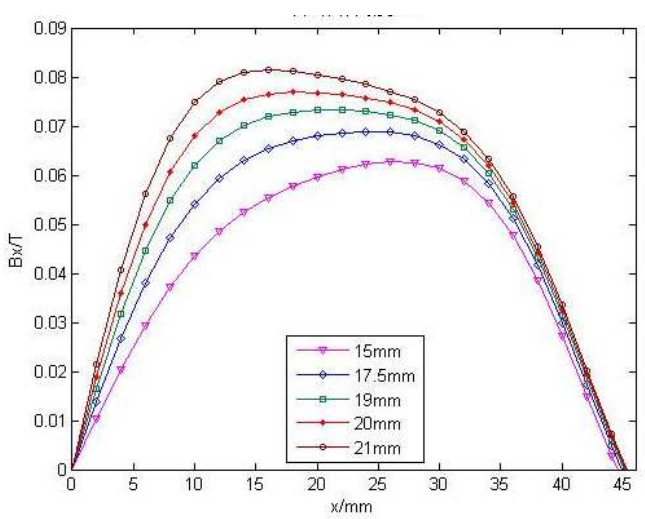

(a) inner magnets

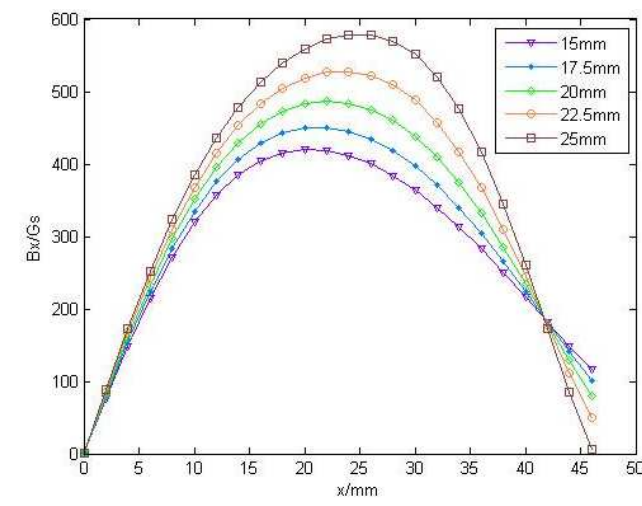

(b) outer magnets

Fig. 7. Simulated distribution curve of horizontal magnetic induction intensity of inner and outer magnets at different heights

After the optimization of target structure, more optimization using the ADPL optimization program in Ansys is made in order to reach optimum distribution of horizontal magnetic induction intensity. Fig. 8 shows the optimization process of objective function (OBT). From the figure, it can be seen that the optimization program has made 35-step iterative optimization and the optimum value appears in the 33th step. Fig.9 shows different distribution of the horizontal magnetic induction intensity on target surface before and after optimization.

After the analysis of magnetic field, researches of dynamic calculation and visualization based on the analysis results of magnetic field are performed. Through secondary development by APDL language, the particles' movement in sputtering process of magnetron sputtering coater is aquired. Finally, using ADAMS (Automatic Dynamic Analysis of Mechanical System) software's strong kinematic and dynamic analysis capabilities and open interfaces and program structure to simulate the movement of charged particles in magnetic field and high frequency alternating electric field of magnetron sputtering coater, thereby achieve visual animation simulation functions of typical magnetron sputtering virtual prototype. 


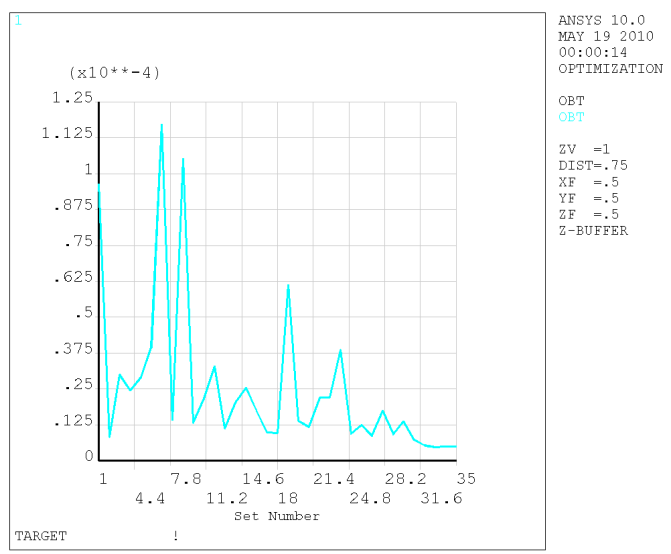

Fig. 8. The optimization process of OBT

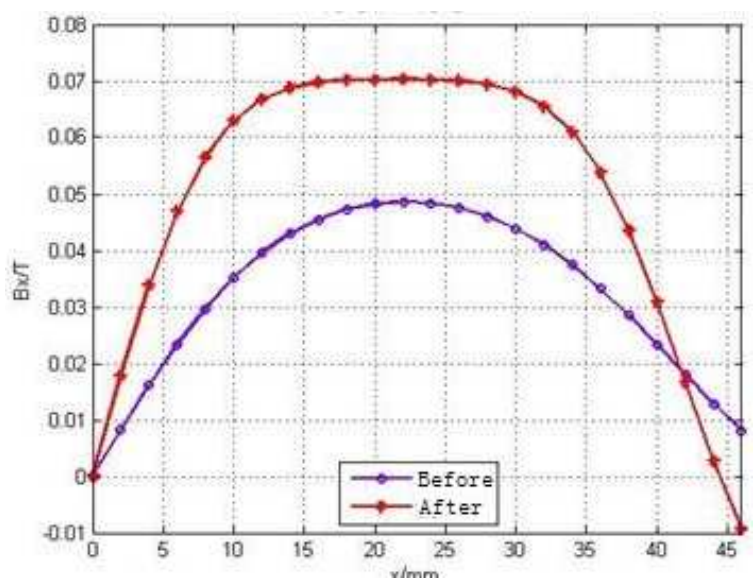

Fig. 9. Contrast of horizontal magnetic induction intensity distribution on target surface before and after optimization

\section{Summary}

Based on deep understanding of virtual prototyping technology and magnetron sputtering coating technology, the framework of a typical cathode target on magnetron sputtering coater is constructed, which provides a theoretical basis for the implementation of cathode target virtual model design.

The virtual models of cathode target including virtual parts and virtual assembly are realized using Solidworks software.

The visual simulation of particles in magnetron sputtering coater virtual model is carried, which explores a way to induct physical process into magnetron sputtering coater design. The visual simulation helps designers to intuitively understand the acceleration process and evaluate the design effect.

The research demonstrates the feasibility of virtual design and visual simulation technology on vacuum coater design.

\section{References}

[1] H. Zou. Design Principles of Mechanical System, China Science Press, Beijing, 2003.

[2] T.Y. Xiao, X. L. Han. Research On Infrastructure of Virtual Manufacturing Technology, Computer Integrated Manufacturing System-CIMS. 5 (1999) 56-59.

[3] C. Shukla, M. Vazquez, F. F. Chen. Virtual manufacturing: An pverview, Computers and Industrial Engineering. 21(1996) 79-82.

[4] T. Y.Xiao. The Progresses and Prospects of the Research on Virtual Manufacturing. Joural of System Simulation. 9(2004) 1879-1913.

[5] K.Y. Shi, R. H.Tan, Q. Li, et al. Research of virtual design platform based on SolidWorks, Machinery Design and Manufacture. 4(2009) 72-74.

[6] S. H. Wang, D. L. Wang, Y. M. Li. Using Software ANSYS to Analyze Electromagnetic Process, HIGH VOLTAGE APPARATUS.3(2002) 27-33.

[7] J. M. Jin. Electromagnetic Field Finite Element Method, China Xi'an Electronic Science and Technology University Press, Xi’an,2001.

[8] L. C. Zhang, S. Xu. Finite Element Method in Electromagnetic Computation, China Railway Publishing House, Beijing, 1996 